\title{
Quantum issues with structured light
}

\author{
Mathew D. Williams, David S. Bradshaw and David L. Andrews* \\ School of Chemistry, University of East Anglia, Norwich NR4 7TJ, United Kingdom
}

\begin{abstract}
Descriptions of optical beams with structured wavefronts or vector polarizations are widely cast in terms of classical field theory. The corresponding fully quantum counterparts often present new insights into what is physically observed, and they are especially of interest when tackling issues such as entanglement. Similarly, when determining angular momentum densities, it appears that the separate roles of photon spin and beam topological charge can only be satisfactorily addressed within a quantum framework. In some such respects, the quantum versions of theory might be considered to introduce an additional layer of complexity; in others, they can clearly and very substantially simplify the theoretical representation. At the photon level, the fully quantized descriptions of topologically structured and singular beams nonetheless raise important fundamental questions and puzzles, whose resolution continue to invite attention. Many of the mechanistic interpretations and predictions (those that appear to be supported by a true congruence between classic and quantum optical descriptions, essentially conflating electromagnetic field and state wavefunction concepts) can lead to theoretical pitfalls. This paper highlights some physical implications that emerge from a fully quantum treatment of theory.
\end{abstract}

Keywords: structured light, optical vortex, twisted beam, vector polarized light, quantum optics, singular light, topological light, nanophotonics.

\section{INTRODUCTION}

Our increasing dependence on telecommunication necessitates new and improved methods for efficient data transfer. As a result, the suitability and future prospects of current methods are under greater than ever scrutiny. The polarization states of Gaussian light offer a binary basis, essentially one bit per photon. One possibility for achieving a marked improvement in data transmission is to utilize structured light. The potential for such light to convey more information than Gaussian light is held within a 'twist' that is applied to the optical wavefront. 'Structured light' is, in fact, an umbrella term that encompasses many families of intensity, phase and polarization topology that in principle has the benefit of having noninterference between each mode. Such beams are generally produced by introducing a wavefront modification, usually applied to a conventional Gaussian beam, and achieved by passage through a number of optical elements. ${ }^{1-5}$ The device that offers the widest variety of radiation mode structures is a spatial light modulator ${ }^{6,7}$ which has the boon of dynamic control over the pixel formation and resultant transmission structure. The form of structured light that has been most widely studied is the Laguerre-Gaussian (LG) mode, wherein each photon is capable of conveying an angular momentum that is not associated with spin character. ${ }^{8}$

Many recent articles concerning such twisted beams suggest a capacity for one or two orders of magnitude increase in data transfer rate. ${ }^{9-12}$ Such aspirations reflect the fact that topological charges with a magnitude of more than 100 have been reported to date. ${ }^{13}$ Moreover, the grounds for such advances in communication are not limited to simple photon transfer, but may also exploit quantum entanglement between separate photons, with recent work achieving the entanglement of a second degree of freedom: the orbital angular component in addition to the spin state. ${ }^{14,15}$ 
At the photon level, the fully quantized descriptions of topologically structured and singular beams in fact raise important fundamental questions and puzzles, whose resolution continue to invite attention. It is not simply the notion of a photon travelling along a path ostensibly longer than a straight line (as several recent papers have suggested ${ }^{16}$ ) that offers a conundrum. For example, can a single photon can with high fidelity convey the entire structure of a full beam? Here, we highlight the quantum features intrinsic to a photon in the analysis of each aspect of the structured light. The context is that methods similar to those involved in optical vortex production can be deployed to detect a specific topological charge ${ }^{17}$ - the detection process is fundamentally subject to the same principles as its time inverse counterpart for emission, which has been addressed in several publications. ${ }^{18-20}$

The next section begins our analysis by introducing a new expression for the fundamental photon commutation relation, taking particular heed to the range of individual characteristics each photon carries; the analysis subsequently incorporates LG light and the associated additional photon degrees of freedom. The following, third section continues by considering the commutation relations - specifically the photon number-phase commutator and the associated uncertainty principle. Finally, the combined implications of all these propositions are considered in a discussion section, which addresses their context within the current literature.

\section{ORTHOGONAL MODES AND QUANTUM COMMUTATION}

Within the purview of quantum optics, photons are most often regarded as quanta associated with specific radiation modes. Designating those modes with labels $l, l^{\prime}$ etc., the boson character of these quanta is exhibited through the associated canonical commutation relations for the photon annihilation operator $a_{l}$, and counterpart creation operator $a_{l^{\dagger}}^{\dagger}$. The general relation is commonly displayed in Kronecker delta form:

$$
\left[a_{l}, a_{l^{\prime}}^{\dagger}\right]=\delta_{l l^{\prime}}
$$

However, the full quantum depiction formally requires a further sub-division of these modes according to their wavevector $\mathbf{k}$ and polarization state, the latter denoted by secondary labels $\eta$ and $\eta^{\prime}$. This introduces another level of restriction on the commutation relations: ${ }^{21}$

$$
\left[a^{(\eta)}(\mathbf{k}), a^{\dagger\left(\eta^{\prime}\right)}\left(\mathbf{k}^{\prime}\right)\right]=\delta_{\mathbf{k k}^{\prime}} \delta_{\eta \eta^{\prime}}
$$

Nonetheless, although the latter form is more accurate, both representations are somewhat flawed. The problem is most readily identified in the latter, in which the form of the first delta is improper, as it contains a pair of indices that do not refer to an orthogonal basis. More correctly, this incorrect commutation relation can be correctly recast in a form suitable for the limiting case of an infinite plane wave mode using a Dirac delta function, as follows;

$$
\left[a^{(\eta)}(\mathbf{k}), a^{\dagger\left(\eta^{\prime}\right)}\left(\mathbf{k}^{\prime}\right)\right]=\left(8 \pi^{3} V\right)^{-1} \delta^{3}\left(\mathbf{k}-\mathbf{k}^{\prime}\right) \delta_{\eta \eta^{\prime}}
$$

where $V$ is the quantization volume, and the single Kronecker delta in equation (3) is fully restricted to orthogonal basis sets - such as might be represented by any pair of points on opposite sides of a Poincaré sphere. (In passing we note that it is this orthogonality of basis polarization states that represents the usual binary basis for conveying photon information.) The constants on the right-hand side of equation (3) are derived to satisfy the dimensionless character of the commutator. 
For structured laser light, which has a specific direction of propagation, equation (3) is best cast in cylindrical coordinates:

$$
\left[a^{(\eta)}(\mathbf{k}), a^{\dagger\left(\eta^{\prime}\right)}\left(\mathbf{k}^{\prime}\right)\right]=\left(8 \pi^{3} k V\right)^{-1} \delta\left(k_{z}-k_{z}^{\prime}\right) \delta\left(k_{r}-k_{r}^{\prime}\right) \delta\left(\phi_{\mathbf{k}}-\phi_{\mathbf{k}^{\prime}}\right) \delta_{\eta \eta^{\prime}}
$$

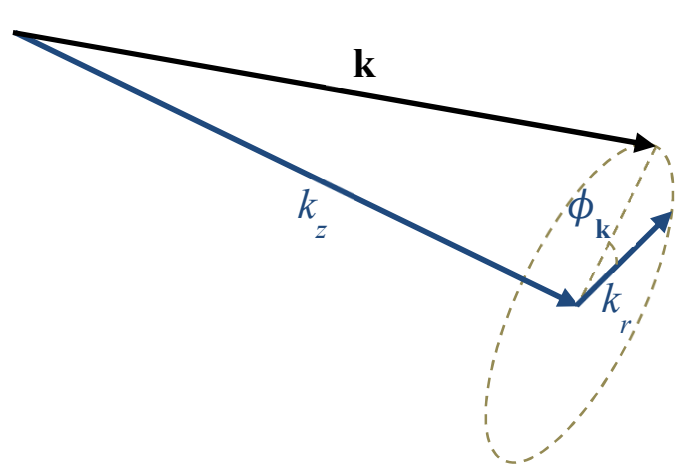

Figure 1. A representation of the wavevector $\mathbf{k}$ for light with a structured aspect in terms of axial and radial components: $k_{z}$, and $k_{r}$ respectively, and an azimuthal angle $\phi_{k}$.

In equation (4), the product of four delta functions signifies that if two radiation modes differ in any one or more of the parameters $k_{z}, k_{r}, \phi_{\mathbf{k}}$ and $\eta$, they are orthogonal - and therefore have the capacity to convey independent information. However, it has to be recognized that precise modal orthogonality cannot always be secured - at least as a result of experimental limitations, but also conceivably for more fundamental quantum uncertainty reasons to be discussed in a later section. To reflect this, it is appropriate to recast the longitudinal wave-vector constraint in terms of a limit. There are various choices available for representing the Dirac delta function in such a form. For our present purposes - and truest to the physicality of beam propagation - we choose the Lorentzian form, effectively representing the extent of local divergence between beams with axial wave-vector components $k_{z}$ and $k_{z}^{\prime}$;

$$
\delta\left(k_{z}-k_{z}^{\prime}\right)=\frac{1}{\pi} \lim _{\varepsilon \rightarrow 0}\left[\frac{\varepsilon}{\left(k_{z}-k_{z}^{\prime}\right)^{2}+\varepsilon^{2}}\right] .
$$

Here, a nonzero $\varepsilon$ indicates a degree of walk-off from the beam wave-vector: specifically, it is the HWHM (half-width at half-maximum) linewidth of the distribution describing the wave-vector offset.

We now turn to consider LG light in particular. Here, the photon creation and annihilation operator commutation relation emerges as:

$$
\left[a_{l, p}^{(\eta)}(k \hat{\mathbf{z}}), a_{l^{\prime}, p^{\prime}}^{\dagger\left(\eta^{\prime}\right)}\left(k^{\prime} \hat{\mathbf{z}}\right)\right]=\left(8 \pi^{3} k V\right)^{-1} \delta\left(k_{z}-k_{z}^{\prime}\right) \delta_{l l^{\prime}} \delta_{p p^{\prime}} \delta_{\eta \eta^{\prime}}
$$

The azimuthal and radial functions are now separable, and within the paraxial approximation the radial distribution takes the following form; 


$$
f_{l, p}(r)=\frac{C_{|l|}^{p}}{w_{0}}\left[\frac{\sqrt{2} r}{w_{0}}\right]^{|l|} \exp \left(\frac{-r^{2}}{w_{0}^{2}}\right) L_{l \mid l}^{p}\left(\frac{2 r^{2}}{w_{0}^{2}}\right)
$$

where, $C_{|l|}^{p}$ is the constant arising during the process of normalization, $w_{0}$ is the Gaussian beam-waist for light of topological charge $\pm l$; this expression also introduces a radial index, $p .^{22}$ The main features present are a product of a Gaussian with an associated Laguerre polynomial $L_{l}^{p}(x)$, the latter being one of the solutions of the following partial differential equation:

$$
x \frac{\partial^{2} L_{l}^{p}(x)}{\partial x^{2}}+(p+1-x) \frac{\partial L_{l}^{p}(x)}{\partial x}+l L_{l}^{p}(x)=0 .
$$

Note that it is the modulus of $l$ that appears in equation (7), signifying that optical vortices of opposite topological charge have identical radial distributions; their physical differences arise through their phase factors, considered below.

Orthogonality amongst the associated Laguerre polynomials is usually specified with respect to those of the same index $p$. The form that is relevant for Laguerre-Gaussian beam applications, which also introduces a weighting factor, is expressible as follows;

$$
\int_{0}^{\infty} e^{-x} x^{p} L_{l}^{p}(x) L_{l^{\prime}}^{p}(x) d x=\frac{(l+p) !}{l !} \delta_{l l^{\prime}}
$$

More generally, ${ }^{23}$ it may be noted, for $\Re(\mu)>-1 ; m, n \in \mathbb{N}_{0}$ the following in terms of hypergeometric functions is found;

$$
\int_{0}^{\infty} x^{\mu} e^{-x} L_{l}^{p}(x) L_{l^{\prime}}^{p^{\prime}}(x) d x=\left(\begin{array}{c}
l+p \\
l
\end{array}\right)\left(\begin{array}{c}
l^{\prime}+p^{\prime}-\mu-1 \\
l^{\prime}
\end{array}\right){ }_{3} F_{2}\left(-m, \mu+1, \mu-p^{\prime}+1 ; p+1, \mu-p^{\prime}-n+1 ; 1\right) \Gamma(\mu+1) .
$$

We are now in a position to establish the vector potential for a Laguerre-Gaussian mode, i.e..$^{8}$

$$
\mathbf{a}^{\perp}(\mathbf{r})=\sum_{k, \eta, l, p}\left(\frac{\hbar}{\varepsilon_{0} c k V}\right)^{1 / 2}\left\{\mathbf{e}^{(\eta)}(k \hat{\mathbf{z}}) a_{k, \eta, l, p} f_{l, p}(r) e^{i(k z+l \phi)}+\overline{\mathbf{e}}^{(\eta)}(k \hat{\mathbf{z}}) a_{k, \eta, l, p}^{\dagger} \bar{f}_{l, p}(r) e^{-i(k z+l \phi)}\right\} .
$$

First, it is observed that the mode sum is taken over four parameters, essentially signifying four degrees of freedom. These contrast with the usual four degrees of freedom that feature in a conventional plane wave expansion, namely three for the three Cartesian components of the wave-vector, and one for the polarization state. The difference is an important one, forming the basis for the conveyance of additional information per photon. It is also worth pointing out that the index $p$ has to be included in the summation in equation (11), for generality and completeness of the modal decomposition. ${ }^{24}$ However in applications seeking to exploit mode orthogonality, then for a given $k, \eta$, it is sufficient to consider only the $l$ values to be different, and hence $p$ is often ignored, or not discriminated, or simply assumed to be 0 . Recently, however, it has been acknowledged that index $p$ is more important than initially thought and, as a result, its role has seen a growth in interest. ${ }^{25,26}$ The $e^{i l \phi}$ factor in the analytic signal for LG modes is consistent with the orthogonality of modes with different topological charge, reflected in the $\delta_{l^{\prime}}$ factor in the photon commutation relation. It is the orthogonality of disparate modes - through either their radial or angular functions, or both - that offers the basis for determining the requisite information content in a detected beam. 
(a)

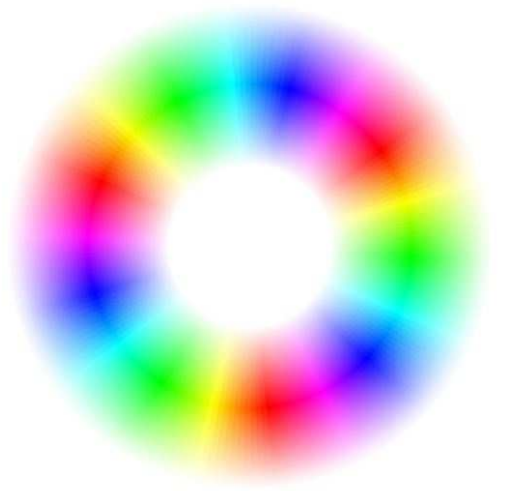

(b)

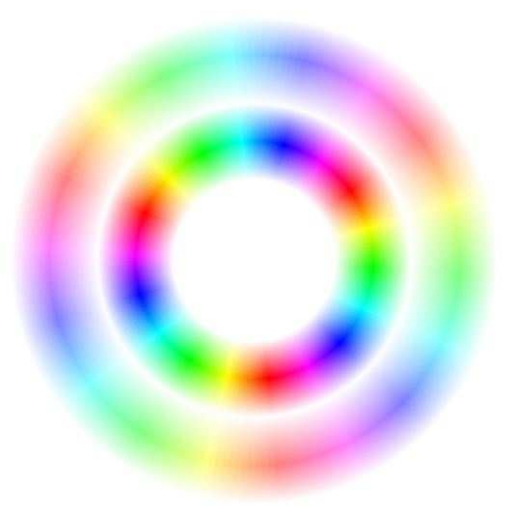

Figure 2. Phase map with superimposed intensity distribution for $(a) l=3, p=0$ and $(b) l=3, p=1$. Sweeping circularly around either simulation, for a given radial position, the phase cycles through $6 \pi^{c}$.

Although methods have been devised to sort optical modes on the basis of their orbital angular momentum (OAM), such that each mode can confidently be sorted from ten or more other $l$ values, incomplete modal orthogonality for different $l$ values is evident in the results of most experiments. ${ }^{27,28}$ Imperfections are also apparent in recent experiments on the multiplication of OAM values. ${ }^{29}$ These imperfections could be arising from experimental error; however, we anticipate a fundamental limit of quantum character, which cannot be crossed.

Figure 3 provides a representation for the evolution of a beam with an LG nature; tracing the progression of phase (one is seen in Figure 2) through a series of wavefront cross-sections, three helical surfaces of constant and equivalent phase can be identified.

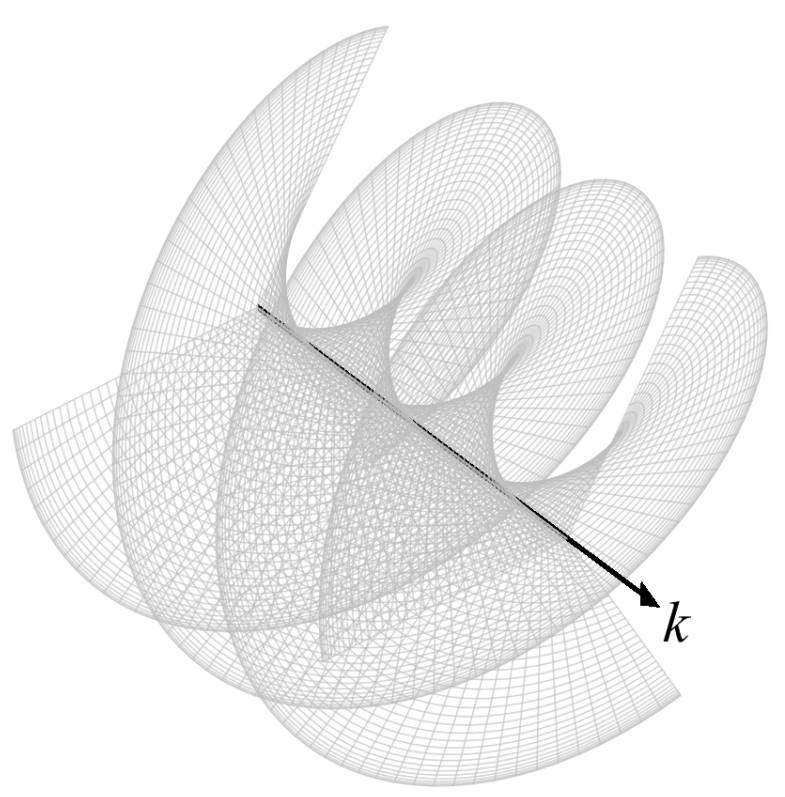

Figure 3. Procession in wavefront for an LG beam with $l=3$ over the course of three wavelengths, depicted as three interleaved helices, each representing a surface of constant phase. 


\section{QUANTUM OPTICAL PHASE ISSUES}

The stochastic nature of any real radiation source gives a distribution of photon numbers, and associated photon statistics. A distribution of phase also arises, leading to beam properties such as degrees of coherence that are meaningless for individual photons. Quantum phases are relative - system wavefunctions are arbitrarily multiplicable by a universal phase factor, with the dynamics unchanged - and only become meaningful when registered as a difference. Before the mid1980s, it had been widely believed that no Hermitian form of operator for optical phase, $\vartheta$, existed. This was understood on the basis of assuming a connection between forms such as $\widehat{e^{-i \vartheta}}$ and $\widehat{e^{i \vartheta}}$ with the photon creation and annihilation operators, accounting for their non-commutativity. One widely accepted solution to this surprisingly taxing problem was proposed by Pegg and Barnett, ${ }^{30,31}$ involving a result of the same form as the earlier Susskind-Glogower operators, ${ }^{32}$ except that the behavior of the phase operator is converted from 'ladder' to 'cycle'. Explicitly, in an arbitrarily limited $(s+1)$ dimensional Fock space, this operator is given by;

$$
\widehat{e_{\vartheta} \phi}=|0\rangle\langle 1|+| 1\rangle\langle 2|+\ldots+| s-1\rangle\left\langle s\left|+e^{i(s+1) \vartheta_{0}}\right| s\right\rangle\langle 0|
$$

where $s$ is the highest number state in a series and the final term gives the cyclic behavior to the operator. (Issues arise in taking the formal limit as $s \rightarrow \infty$, as discussed in more detail elsewhere ${ }^{30}$ ). An alternative derivation of the Pegg-Barnett phase operator, which involves applying a canonical transformation to the action and phase-angle variables of a harmonic oscillator, is also found in the literature. ${ }^{33}$ So far it remains not entirely clear as to whether there exists a completely wellbehaved definition for a phase operator, upon which to build a commutator with the photon number operator. However, one defensible form of commutation with the photon number operator is ${ }^{34}$

$$
\left[\hat{n}, \widehat{e^{i \vartheta}}\right]=-\widehat{e^{i \vartheta}}
$$

From this relationship, we observe that the Hermitian phase operator, $\hat{\vartheta}$, does not commute with the number operator, $\hat{n}$. In specific connection with LG modes, the expectation value of the phase operator is, from equation $(11),(k z+l \phi)$.

The commutation relation (13) can be understood as the formal basis for an uncertainty relation between photon number and optical phase. There has, in fact, been many attempts to formulate a number-phase uncertainty relation from Dirac onwards, but a clear statement appears in Heitler's authoritative 1954 text, expressed as: ${ }^{35}$

$$
\Delta n \Delta \vartheta \geq \frac{1}{2}
$$

More specifically, it follows from Heisenberg's uncertainty principle that the variances in the corresponding properties represented by operators $\hat{\vartheta}$ and $\hat{n}$ must satisfy: ${ }^{36}$

$$
\left\langle\Delta \hat{n}^{2}\right\rangle\left\langle\Delta \hat{\vartheta}^{2}\right\rangle \geq \frac{1}{4}|\langle[\hat{n}, \hat{\vartheta}]\rangle|^{2}
$$

As a result of this interplay of uncertainty it proves difficult to measure optical phase, the fundamental uncertainty convincingly established by Beck et al. in 1993. ${ }^{37}$ Nonetheless, various ingenious techniques have been suggested in the attempt to identify at least an approximate value for optical phase, ${ }^{38-40}$ and one of them has achieved notable success. ${ }^{41}$ 
We now turn to examine the commutation relation of equation (13) in more detail. Once again, the simplicity of its form belies some important detail. Most of the theoretical analysis on this topic assumes there is just one optical mode of interest. However, there is a distinction to be made from quantum optical commutators such as the following; ${ }^{21}$

$$
\left[e_{i}(\mathbf{r}), b_{j}\left(\mathbf{r}^{\prime}\right)\right]=\frac{i \hbar}{\varepsilon_{0}} \varepsilon_{i j k} \frac{\partial}{\partial \mathbf{r}_{k}^{\prime}} \delta\left(\mathbf{r}-\mathbf{r}^{\prime}\right),
$$

in which each of the operators within the commutator carries an implied mode summation over wave-vectors $\mathbf{k}$, so that the whole is to be understood as an $\mathbf{r}$-space representation. In fact, the converse applies to the commutation relation equation (13); the number operator is clearly meaningless without reference to a specific mode, and the observation therefore extends to the left-hand side of equation (13) as a whole. In other words, this commutator relates to a $\mathbf{k}$-space representation of modes that extend over all space $\mathbf{r}$. Thus the left-hand side requires reference to a specific mode, which in turn means the right-hand side must too. It is therefore clear that the optical phase operator must also be mode-specific. This throws new light on the physical meaning, and the limitations of such meaning, that can be attached to optical phase. Clearly, it is associated with a radiation state rather than representing a property of any individual photon. Moreover it does not signify a relative phase between individual photons, but instead denotes a relative phase between the radiation state vectors. The phase operator does not detail a photon phase at a specific point in space, but rather the entire mode's structure. Equally, the number-phase uncertainty product applies individually to each mode of an orthogonal set.

\section{DISCUSSION}

The detection of a structured optical wave-front is the basis for the many emerging applications in imaging, ${ }^{42-46}$ and data transmission, ${ }^{47,48}$ notwithstanding the fact that some of the issues are now coming under scrutiny ${ }^{49}$ Such methods usually require a modal decomposition to sort out each orthogonal mode comprising the beam. Attempting to maximize the information density (and resolution), by exploiting a large number of orthogonal modes delivering the same total intensity, leads to low numbers of photons per mode. As a result of this, a degradation of modal orthogonality can be seen to become more prominent, and as such the beam characterization suffers. Taking the lower limit of mode occupancy there is a fundamental limit at which point it becomes necessary to discriminate between modes; with the ultimate limit, where modal structure confers no clear advantage, most obviously apparent when the mode occupancy is restricted to a binary basis: 0 and 1, i.e. $\Delta n_{k, \eta, l, p} \ll 1$, at which point the optical phase is indeterminate. Of course, with higher photon flux it would matter less; with 100 photons present the phase uncertainty for the same mode detection would be negligible. Contrary to the lessons of 'Young's slits' plane-wave experiments at low intensities, ${ }^{50,51}$ single-photon optics with structured light cannot be entirely identified with beam or ray optics.

Thus, whereas the principle of achieving heightened content at lower intensities can be exploited for data transmission, imaging and adaptive optics, it is conceivable that at some point a trade-off will be met, where increasing the topological charge ceases to deliver an advantage. Explicitly, more highly structured light at low intensities suffers more strongly the consequences of photon number-phase uncertainty, compromising complete mode orthogonality. The ultimate limit, where modal structure confers no clear advantages, is when the number of photons per mode drops to an essentially binary basis, a mode occupation number of 0 or 1 . Here, any conventional detection method will most strongly suffer the consequences of photon number-phase uncertainty, compromising the complete mode orthogonality that is necessary for complete fidelity in transmission and detection. This represents a fundamental limitation on the information conveyable by a single photon. 


\section{ACKNOWLEDGEMENTS}

The authors would like to thank the University of East Anglia and EPSRC for funding this research.

\section{REFERENCES}

[1] Bazhenov, V. Y., Vasnetsov, M. and Soskin, M., "Laser beams with screw dislocations in their wavefronts," Jetp Lett 52, 429-431 (1990).

[2] Bazhenov, V. Y., Soskin, M. and Vasnetsov, M., "Screw dislocations in light wavefronts,” J. Mod. Opt. 39, 985990 (1992).

[3] Beijersbergen, M. W., Coerwinkel, R. P. C., Kristensen, M. and Woerdman, J. P., "Helical-wave-front laser-beams produced with a spiral phaseplate," Opt. Commun. 112, 321-327 (1994).

[4] Marrucci, L., Manzo, C. and Paparo, D., "Optical spin-to-orbital angular momentum conversion in inhomogeneous anisotropic media," Phys. Rev. Lett. 96, 163905 (2006).

[5] Sun, J., Zeng, J. and Litchinitser, N. M., "Twisting light with hyperbolic metamaterials," Opt. Express 21, 1497514981 (2013).

[6] Heckenberg, N. R., McDuff, R., Smith, C. P. and White, A. G., "Generation of optical-phase singularities by computer-generated holograms," Opt. Lett. 17, 221-223 (1992).

[7] Ostrovsky, A. S., Rickenstorff-Parrao, C. and Arrizón, V., "Generation of the "perfect" optical vortex using a liquidcrystal spatial light modulator," Opt. Lett. 38, 534-536 (2013).

[8] Dávila Romero, L. C., Andrews, D. L. and Babiker, M., “A quantum electrodynamics framework for the nonlinear optics of twisted beams,” J. Opt. B: Quantum Semiclass. Opt. 4, S66-S72 (2002).

[9] Lavery, M. P., Roberston, D., Malik, M., Robenburg, B., Courtial, J., Boyd, R. W. and Padgett, M. J., “The efficient sorting of light's orbital angular momentum for optical communications." 85421R-85421R-7.

[10] O'Sullivan, M. N., Mirhosseini, M., Malik, M. and Boyd, R. W., "Near-perfect sorting of orbital angular momentum and angular position states of light," Opt. Express 20, 24444-24449 (2012).

[11] Dudley, A., Schulze, C., Litvin, I., Duparré, M. and Forbes, A., "Quantitatively measuring the orbital angular momentum density of light." 88100E-88100E-7.

[12] Liu, A.-P., Xiong, X., Ren, X.-F., Cai, Y.-J., Rui, G.-H., Zhan, Q.-W., Guo, G.-C. and Guo, G.-P., "Detecting orbital angular momentum through division-of-amplitude interference with a circular plasmonic lens," Sci. Rep. 3, (2013).

[13] Fickler, R., Lapkiewicz, R., Plick, W. N., Krenn, M., Schaeff, C., Ramelow, S. and Zeilinger, A., "Quantum entanglement of high angular momenta," Science 338, 640-643 (2012).

[14] Karimi, E. and Boyd, R. W., "Classical entanglement?," Science 350, 1172-1173 (2015).

[15] Wang, X.-L., Cai, X.-D., Su, Z.-E., Chen, M.-C., Wu, D., Li, L., Liu, N.-L., Lu, C.-Y. and Pan, J.-W., "Quantum teleportation of multiple degrees of freedom of a single photon," Nature 518, 516-519 (2015).

[16] Giovannini, D., Romero, J., Potoček, V., Ferenczi, G., Speirits, F., Barnett, S. M., Faccio, D. and Padgett, M. J., "Spatially structured photons that travel in free space slower than the speed of light," Science, (2015).

[17] Mirhosseini, M., Malik, M., Shi, Z. and Boyd, R., "Efficient separation of the orbital angular momentum eigenstates of light," Nat. Commun. 4, 2781 (2013).

[18] Coles, M. M., Williams, M. D., Saadi, K., Bradshaw, D. S. and Andrews, D. L., "Chiral nanoemitter array: A launchpad for optical vortices," Laser \& Photon. Rev. 7, 1088-1092 (2013).

[19] Williams, M. D., Coles, M. M., Saadi, K., Bradshaw, D. S. and Andrews, D. L., "Optical vortex generation from molecular chromophore arrays," Phys. Rev. Lett. 111, 153603 (2013).

[20] Williams, M. D., Coles, M. M., Bradshaw, D. S. and Andrews, D. L., "Direct generation of optical vortices,” Phys. Rev. A 89, 033837 (2014).

[21] Craig, D. P. and Thirunamachandran, T., [Molecular Quantum Electrodynamics: An Introduction to RadiationMolecule Interactions], Dover Publications, Mineola, NY (1998).

[22] Sonine, N., "Recherches sur les fonctions cylindriques et le développement des fonctions continues en séries," Mathematische Annalen 16, 1-80.

[23] Mavromatis, H. A., "An interesting new result involving associated Laguerre polynomials," International journal of computer mathematics 36, 257-261 (1990).

[24] Schulze, C., Dudley, A., Flamm, D., Duparré, M. and Forbes, A., "Measurement of the orbital angular momentum density of light by modal decomposition," New J. Phys. 15, 073025 (2013). 
[25] Karimi, E., Boyd, R. W., de la Hoz, P., de Guise, H., Řeháček, J., Hradil, Z., Aiello, A., Leuchs, G. and SánchezSoto, L. L., "Radial quantum number of Laguerre-Gauss modes," Phys. Rev. A 89, 063813 (2014).

[26] Plick, W. N. and Krenn, M., "Physical meaning of the radial index of Laguerre-Gauss beams," Phys. Rev. A 92 , 063841 (2015).

[27] Berkhout, G. C., Lavery, M. P., Courtial, J., Beijersbergen, M. W. and Padgett, M. J., "Efficient sorting of orbital angular momentum states of light," Phys. Rev. Lett. 105, 153601 (2010).

[28] Mirhosseini, M., Magaña-Loaiza, O. S., O’Sullivan, M. N., Rodenburg, B., Malik, M., Lavery, M. P. J., Padgett, M. J., Gauthier, D. J. and Boyd, R. W., "High-dimensional quantum cryptography with twisted light," New J. Phys. 17, $033033(2015)$.

[29] Potoček, V., Miatto, F. M., Mirhosseini, M., Magaña-Loaiza, O. S., Liapis, A. C., Oi, D. K. L., Boyd, R. W. and Jeffers, J., “Quantum Hilbert Hotel,” Phys. Rev. Lett. 115, 160505 (2015).

[30] Pegg, D. T. and Barnett, S. M., “Unitary phase operator in quantum mechanics,” Europhys. Lett. 6, 483-487 (1988).

[31] Barnett, S. M. and Pegg, D. T., “On the Hermitian optical phase operator,” J. Mod. Opt. 36, 7-19 (1989).

[32] Susskind, L. and Glogower, J., "Quantum mechanical phase and time operator," Physics 1, 49-61 (1964).

[33] Luis, A. and Sánchez-Soto, L. L., "Alternative derivation of the Pegg-Barnett phase operator,” Phys. Rev. A 47, 1492-1496 (1993).

[34] Loudon, R., [The Quantum Theory of Light], Oxford University Press, Oxford (2000).

[35] Heitler, W., [The Quantum Theory of Radiation], Dover, Mineola, New York (1954).

[36] Vaccaro, J. A. and Pegg, D. T., "Physical number-phase intelligent and minimum-uncertainty states of light," J. Mod. Opt. 37, 17-39 (1990).

[37] Beck, M., Smithey, D. T., Cooper, J. and Raymer, M. G., "Experimental determination of number-phase uncertainty relations," Opt. Lett. 18, 1259-1261 (1993).

[38] Noh, J. W., Fougères, A. and Mandel, L., "Measurement of the quantum phase by photon counting," Phys. Rev. Lett. 67, 1426-1429 (1991).

[39] Wiseman, H. M., "Adaptive phase measurements of optical modes: Going beyond the marginal Q distribution," Phys. Rev. Lett. 75, 4587-4590 (1995).

[40] Barnett, S. M. and Pegg, D. T., "Phase measurement by projection synthesis," Phys. Rev. Lett. 76, 4148-4150 (1996).

[41] Armen, M. A., Au, J. K., Stockton, J. K., Doherty, A. C. and Mabuchi, H., "Adaptive homodyne measurement of optical phase," Phys. Rev. Lett. 89, 133602 (2002).

[42] Liu, C. and Kim, D. Y., "Differential imaging in coherent anti-Stokes Raman scattering microscopy with LaguerreGaussian excitation beams," Opt. Express 15, 10123-10134 (2007).

[43] Planchon, T. A., Gao, L., Milkie, D. E., Davidson, M. W., Galbraith, J. A., Galbraith, C. G. and Betzig, E., "Rapid three-dimensional isotropic imaging of living cells using Bessel beam plane illumination," Nat. Meth. 8, 417-423 (2011).

[44] Iketaki, Y. and Bokor, N., "Super-resolution microscope using a two-color phase plate for generating quasiLaguerre-Gaussian beam," Opt. Commun. 285, 3798-3804 (2012).

[45] Lalithambigai, K., Anbarasan, P. M. and Rajesh, K. B., "Study on higher order azimuthally polarized LaguerreGaussian mode beams with high NA lens," J Theor Appl Phys 8, 1-9 (2014).

[46] Szatkowski, M., Popiołek-Masajada, A. and Masajada, J., "Optical vortex in microscopy imaging.” 9194, 91941D91941D-6.

[47] Bozinovic, N., Yue, Y., Ren, Y., Tur, M., Kristensen, P., Huang, H., Willner, A. E. and Ramachandran, S., "Terabitscale orbital angular momentum mode division multiplexing in fibers," Science 340, 1545-1548 (2013).

[48] Willner, A. E., Huang, H., Yan, Y., Ren, Y., Ahmed, N., Xie, G., Bao, C., Li, L., Cao, Y., Zhao, Z., Wang, J., Lavery, M. P. J., Tur, M., Ramachandran, S., Molisch, A. F., Ashrafi, N. and Ashrafi, S., "Optical communications using orbital angular momentum beams," Adv. Opt. Photon. 7, 66-106 (2015).

[49] Barreiro, J. T., Wei, T.-C. and Kwiat, P. G., "Remote preparation of single-photon 'hybrid' entangled and vectorpolarization states," Phys. Rev. Lett. 105, 030407 (2010).

[50] Jack, B., Padgett, M. J. and Franke-Arnold, S., “Angular diffraction,” New J. Phys. 10, 103013 (2008).

[51] Liu, R., Phillips, D. B., Li, F., Williams, M. D., Andrews, D. L. and Padgett, M. J., "Discrete emitters as a source of orbital angular momentum," J. Opt. 17, 045608 (2015).

*david.andrews@physics.org 